\title{
Reflektierte Integration - Transkulturelle Denkbewegungen
}

\author{
Transculturality as a Paradigm for Intercultural Approaches in Psychiatry and \\ Psychotherapy
}

Autor

Institut
Ernestine Wohlfart

Klinik für Psychiatrie und Psychotherapie, Charité - Universitätsmedizin Berlin, Campus Charité Mitte

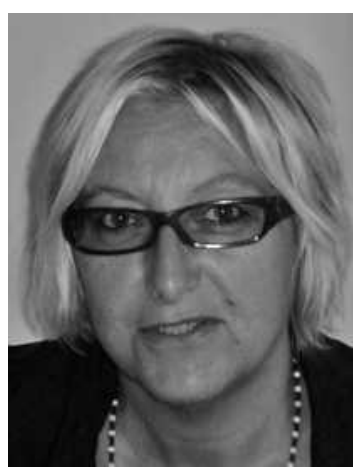

Dr. med. Ernestine Wohlfart
Bibliografie

DOI $10.1055 / \mathrm{s}-2007-986184$

Psychiat Prax 2007; 34:

317-319 @ Georg Thieme

Verlag KG Stuttgart · New York . ISSN 0303-4259

Korrespondenzadresse Dr. med. Ernestine Wohlfart Zentrum für Interkulturelle Psychiatrie, Psychotherapie und Supervision (ZIPP), Klinik für Psychiatrie und Psychotherapie, Charité - Universitätsmedizin Berlin, Campus Charité Mitte Charitéplatz 1

10117 Berlin

ernestine.wohlfart@charite.de
Der Beitrag basiert auf Praxiserfahrungen, Forschung und Theorieentwicklungen zu transkulturellen Ansätzen in der psychiatrischen und psychotherapeutischen Behandlung am ZIPP1.

\section{Interkulturalität und der Anspruch an interkulturelle Öffnung von psychi- atrischen/psychotherapeutischen Institutionen $\nabla$}

In Anbetracht einer zunehmenden internationalen Klientel kann es nicht der moralisch korrekte Anspruch einiger Weniger sein, die im psychologischen, psychiatrischen Feld tätig sind, sich zu öffnen und interkulturelle Kompetenzen [1] zu entwickeln. Es ist vielmehr notwendig, eine Professionalisierung zu erzielen, die neue Erkenntnismöglichkeiten im Umgang mit kultureller Differenz ermöglicht [2-4]. Das bedeutet, die gesellschaftlichen Veränderungen und Wanderungen zu kennen und zu reflektieren. Migrationsbedingte und kulturdynamische/transkulturelle Konflikte sollten als Ursachen für psychische Erkrankungen erkannt und anerkannt werden. Es gilt wahrzunehmen, dass man es notwendigerweise auch in Institutionen der Regelversorgung mit Individuen zu tun hat, die aus unterschiedlichen kulturellen Kontexten kommen und deren subjektive Interpretation von Krankheit nicht unbedingt der euroamerikanischen entsprechen muss. Es erscheint wichtig, darüber nachzudenken, was es für unser professionelles Handeln bedeutet, wenn wir mit einem Teil unserer Patien-

\footnotetext{
${ }^{1}$ ZIPP-Zentrum für interkulturelle Psychiatrie, Psychotherapie und Supervision, Charité, Campus Mitte. Das ZIPP gliedert sich in 3 Bereiche: I. = Interdisziplinäre, interkulturelle Forschungsgruppe; II. = Ethnopsychiatrische Ambulanz integriert in die Institutsambulanz der Klinik für Psychiatrie und Psychotherapie am Campus Charite Mitte; III. = Weiterbildungsbereich/interkulturelle Supervision.
}

ten nicht mehr in einem gemeinsamen Bedeutungszusammenhang stehen, auf den wir in der Diagnosestellung und Behandlung (unbewusst) rekurrieren können.

Es finden interkulturelle Begegnungen statt, die notwendigerweise eine erhöhte Spannung bei den Beteiligten erzeugen; eine Spannung zwischen dem fremden Anderen und dem eigenen Vertrauten, welche allzu häufig nicht wahrgenommen werden darf und deshalb in Stereotypenbildung mündet, um die eigene professionelle Hilflosigkeit des Nichtverstehens aushaltbarer zu machen. Diese spiegeln sich wider in ethnisierenden Zuschreibungen, Fehldiagnosen oder einer übertriebenen Pathologisierung [5] des fremden Patienten. Als Beispiel können das lange kursierende „Ganzkörperschmerzsyndrom“ (auch „Mittelmeersyndrom“ genannt) oder akute Erregungszustände im Rahmen neurotisch unlösbarer Konflikte genannt werden, die als vermeintliche schizophrene Psychose gedeutet werden. Das Konzept eines kultursensitiven Ansatzes entstand, wie Scharfetter und Bridler aufzeigen, in Zusammenhang mit den eingangs genannten Notwendigkeiten zur interkulturellen Kommunikation, die alle am Prozess Beteiligten dann überfordert, wenn die Situation nicht als Chance zur Veränderung begriffen wird. Allzu oft wird die Differenz und die Fremdheitserfahrung in interkulturellen Situationen von den Experten und Therapeuten nicht wahrgenommen und die westliche Prägung wesentlicher Erklärungsmodelle psychischer Krankheit (wie z.B. die Annahme einer Trennung zwischen Körper und Psyche) wird nicht ausreichend wahrgenommen. Die Therapeuten und das psychiatrische Versorgungssystem werden als vermeintlich kulturfrei verstanden und die Einbettung in soziokulturelle Kontexte wird übersehen. Nur die Anderen haben dann eine „Kultur“ und werden in ihren subjektiven Erklärungsmodellen von dieser bestimmt. Hilflosigkeit, Unsicherheit und Gefühle der Über- 
forderung bei der Diagnosestellung, die in interkulturellen Situationen auftreten können, werden hierbei oft nicht zugelassen und thematisiert. Vielmehr sollen wir Therapeuten hohen Idealen entsprechen und den Bedürfnissen der Patienten tolerant und flexibel entgegenkommen. So richtig diese Ansprüche für eine demokratische Gesellschaft sind, so unscharf sind sie für die Praxis. Zum Verständnis der eigenen Position gehört auch die Reflektion der zeitgenössischen wie unserer persönlichen und politischen Haltung zu Migration und „Ausländern“, welche die Begegnung mit den Anderen, mit den Fremden beeinflusst. Je nach eigenen Erfahrungen auch mit Fremdheitsgefühlen [6] werden wir in der Lage sein die benannten Spannungen und das Nichtverstehen in interkulturellen Begegnungen auszuhalten. Deshalb wird eine rein institutionell verordnete interkulturelle Kompetenz nicht nachhaltig wirksam werden können. Sie wird weder den Patienten das Gefühl vermitteln, in ihrem Andersein angenommen zu werden, noch die Behandler in die Lage versetzen, ihr eigenes Konzept von psychischen Erkrankungen zu reflektieren und Diversität wahrzunehmen. Um diese Ziele zu erreichen, sollte die transkulturelle Kommunikation und Psychiatrie bereits in der universitären Ausbildung und in verbindlichen Weiterbildungsangeboten verankert werden.

\section{Assimilation - Inklusion - Inkorporation \\ $\nabla$}

Für die weitere Argumentation „PRO Integration von Migranten in die Regelversorgung“, möchten wir uns einen Schritt aus dem klinischen Alltag entfernen und die gesellschaftlichen Rahmenbedingungen und Diskurse bezüglich Integration und Migration betrachten. Dies ermöglicht es, die gängige gesellschaftliche Perspektive zu reflektieren, die uns nicht immer bewusst prägt, aber die Entwicklung von interkulturellen Ansätzen in Forschung wie Klinik maßgeblich beeinflusst.

Besonders westeuropäische Länder transformieren sich derzeit zu Einwanderungsgesellschaften. Dies wird von politischer Seite jedoch nicht explizit anerkannt. Zuwanderer haben hohe Hürden zu überwinden, häufig verlieren sie ihren sozialen Status, den sie im Herkunftsland hatten und kommen ganz unten an. Noch immer wird die gesellschaftliche Integrationsdebatte maßgeblich von Assimilationstheorien bestimmt, die u.a. auf den Soziologen Esser [7,8] zurückgehen. Diesen Konzepten liegt ein Bild von nationalen, als kulturell homogen verstandenen Gesellschaften (und Kulturen) zugrunde und eine erfolgreiche Integration hat die Anpassung der Migranten an die Aufnahmegesellschaft zum Ziel. Das darin enthaltene Potenzial des Scheiterns verbleibt bei den Migranten. Es gibt Inländer und Ausländer, die im gleichen Land leben.

Um eine nachhaltige interkulturelle Kompetenz auch im Gesundheitssystem zu etablieren, erscheint es demgegenüber als notwendig, neueren soziologischen Debatten zu folgen, die andere Integrationsansätze formulieren. Denn diese Diskurse eröffnen neue gesellschaftliche Perspektiven auf die veränderlichen Parameter, welche die internationale Migration beeinflussen.

Sen, Halm und Sauer [9] eröffnen mit ihrem Konzept der Inklusion ein Integrationskonzept, welches im Gegensatz zur einseitigen Anpassung der Migranten an die Aufnahmegesellschaft den Migranten eine Doppelorientierung ermöglicht: „Die Aufnahmegesellschaft gewährt den Migranten gleiche Teilhabechancen und ermöglicht gleichzeitig aber auch weiterhin die Orientierung an ihrer Herkunftskultur. Integration ist hierbei als ein wechselseitiger Vorgang verstanden, der Anstrengungen von Deutschen und Zuwandern gleichermaßen fordert" [9]. Eine solche Perspektive erscheint vielversprechend zum Abbau von Segregation, Ghettoisierung, Ausgrenzung und der damit verbundenen Weiter- bzw. Neuentwicklung von Stereotypen.

Die Autoren Pries und Goebel [10] gehen mit ihrem Inkorporationskonzept noch einen Schritt weiter und verstehen unter Integration ein ergebnisoffenen Prozess, welcher ein neues Bild von Migranten und Migration ermöglicht. Dieses entstünde, wenn die Perspektive „nationaler“ Container-Gesellschaften aufgebrochen würde und Formen einer partiellen, segmentierten oder multiplen Inkorporation berücksichtigt würden.

Unseres Erachtens, und da stimmen wir Scharfetter und Bridler zu, müsste sich die Flexibilität und Toleranz des Gesundheitswesens gerade auch darin widerspiegeln, mit neuen Ansätzen und Konzepten zu experimentieren

\section{Transkulturelle Denkbewegungen \\ $\nabla$}

In der klinischen und theoretischen Arbeit am ZIPP fokussieren wir diesbezüglich auf einem reflexiven Aushandlungsprozess zwischen dem Eigenen und dem Fremden, sowohl in der jeweiligen klinischen Begegnung als auch in der Weiterentwicklung von wissenschaftlichen Methoden und Behandlungskonzepten, im Austausch mit Professionellen anderer Disziplinen und im interkulturellen Team. Die Basis dieser reflexiven Denk- und Praxisbewegungen ist in ethnopsychiatrischen/ethnopsychoanalytischen Konzepten verortet [11-14].

Kulturelle Differenz wird in diesen Ansätzen nicht minimiert, vielmehr werden neue Dimensionen des Verstehens und Verhaltens erzeugt. Differenz irritiert und macht uns in der Auseinandersetzung selbst zu Fremden, sie zeigt uns den „Anderen in uns“. Sie weckt damit aber auch unsere Neugier und unseren Wunsch, uns selbst und den „Anderen“ besser zu verstehen. Um „cultural bias“ (kulturelle Missverständnisse) in der Diagnosestellung und Behandlung zu vermeiden, wäre es notwendig, an einer transkulturellen Denkbewegung teilzunehmen, welche die Vielfalt von Vorstellungen und Lebenseinstellungen verschiedenster kultureller Kontexte nicht ausschließt, sondern reflektiert.

Die Grundlage eines solchen Verständnisses von Interkulturalität bzw. interkultureller Kompetenz (transkulturelles Denken) können z.B. durch Pilotprojekte wie das Ethnomedizinische Zentrum (Hannover), die ethnopsychiatrische Ambulanz am ZIPP (Berlin) oder das Centre Devereux (Paris) und deren Multiplikatoren in der Regelversorgung erarbeitet werden [15].

Wir beenden unseren Beitrag mit Fragen, die uns bei der psychiatrischen Exploration eines Patienten aus einem anderen kulturellen Kontext als hilfreich erscheinen:

1. Wie ist der Realitätsbezug zu beurteilen? Gibt es für den Patienten und die Herkunftskultur nur eine oder mehrere Welten, z. B.: die der Geister. Wie werden Trance, Zustände der Besessenheit und Entrücktheit im jeweiligen Kontext verstanden?

2. Welcher Stellenwert wird Spiritualität und Religiosität von der Gruppe und vom Einzelnen zugewiesen?

3. Wie werden Privatheit, der öffentliche Raum und die gesellschaftlich akzeptable Beziehungsaufnahme definiert? Mit wem kann wie über etwas gesprochen werden?

4. Wie wird Autonomieentwicklung erlebt und bewertet? In welchem Verhältnis steht die Ablösung von der Primärgrup- 
pe zu Ansprüchen an die Erfüllung einer Rollenerwartung der Primärgruppe?

5. Wie werden Extraversion/Introversion und der Ausdruck von Emotionen im jeweiligen kulturellen Kontext bewertet und akzeptiert? Wird durch aktives Verhalten Achtung gesucht oder kann Mann/Frau durch Zurückhaltung ehrbar erscheinen?

6. Fungiert Schmerz als sprachliche Chiffre für seelisches Leiden und existiert in der Ursprungskultur eine Trennung zwischen Körper und Psyche, wie sie in den westlichen Gesellschaften/ Medizinsystemen häufig vorkommt?

7. Sind Suizid/Selbsttötung tabuisiert mit Implikationen für das Individuum über den Tod hinaus?

8. Welche Erwartung besteht an die Experten? Wird eine passive oder aktive Heilung gesucht?

\section{Literatur}

1 Wohlfart E, Hodzic S, Özbek T. Transkulturelles Denken und transkulturelle Praxis in der Psychiatrie und Psychotherapie. In: Wohlfart E, Zaumseil M (Hrsg): Transkulturelle Psychiatrie - Interkulturelle Psychotherapie. Interdisziplinäre Theorie und Praxis. Heidelberg: Springer Medizin Verlag, 2006

2 Englisch M. Eine interkulturelle Supervisionsmethode auf ethnopsychoanalytischer Grundlage. In: Wohlfart E, Zaumseil M (Hrsg): Transkulturelle Psychiatrie - Interkulturelle Psychotherapie. Heidelberg: Springer Medizin Verlag, 2006

3 Gaitanides S. Qualitätsstandards zur interkulturellen Teamentwicklung. IZA Magazin 2002; 1: 4-7

4 Calliess IT, Machleidt W. Transkulturelle Aspekte bei Persönlichkeitsstörungen. Persönlichkeitsstörungen. Persönlichkeitsstörungen 2003; 7: $117-133$

5 Wohlfart E, Özbek T. Eine ethnopsychoanalytische Kasuistik über das Phänomen der Besessenheit. Psyche-Z Psychoanal 2006; 60: 118 - 130

6 Machleidt W, Heinz A. Psychotherapie mit Migranten. Unveröffentlichtes Manuskript, 2007

7 Esser H. Aspekte der Wanderungssoziologie. Assimilation und Integration von Wanderern, ethnischen Gruppen und Minderheiten. Eine handlungstheoretische Analyse. Darmstadt und Neuwind: Luchterhand, 1980

8 Esser $H$. Integration und ethnische Schichtung. Arbeitspapier Nr. 40 des Mannheimer Zentrums für Europäische Sozialforschung. Mannheim: 2001

9 Goldberg A, Halm D, Sauer M (Hrsg). Migrationsbericht des Zentrums für Türkeistudien 2002. Münster: LIT-Verlag, 2001

10 Goebel D, Pries L. Transnationale Migration und Inkorporation von Migranten. Einige konzeptionell theoretische Überlegungen zu einem erweiterten Verständnis gegenwärtiger Inkorporationsprozesse von Migranten. In: Swiaczny F, Haug S (Hrsg): Migration - Integration Minderheiten. Neuere interdisziplinäre Forschungsergebnisse. Materialien zur Bevölkerungswissenschaft, Heft 107. Wiesbaden: Bundesinstitut für Bevölkerungsforschung, 2003: 35-48

11 Devereux G. Ethnopsychoanalyse. Die komplementaristische Methode in den Wissenschaften vom Menschen. Frankfurt a.M.: Suhrkamp, 1978

12 Parin P. Der Widerspruch im Subjekt. Ethnopsychoanalytische Studien. Frankfurt a. M.: Syndikat, 1978

13 Wulff E. Vom Wahn zur Methode. In: Duerr HP (Hrsg): Die wilde Seele. Zur Ethnopsychoanalyse von Georges Devereux. Frankfurt a. M.: Suhrkamp, 1987: $398-418$

14 Nadig M. Interkulturalität im Prozess - Ethnopsychoanalyse und Feldforschung als methodischer und theoretischer Übergangsraum. In: Lahme-Gronostaj H, Leuzinger-Bohleber M (Hrsg): Identität und Differenz. Zur Psychoanalyse des Geschlechterverhältnisses in der Spätmoderne. Opladen: Westdeutscher Verlag, 2000: 87-101

15 Machleidt W. Migration, Integration und psychische Gesundheit. Psychiat Prax 2005; 32: 55-57

\section{Psychiatrische Praxis}

Sozialpsychiatrie • Klinische Psychiatrie •

Public Mental Health · Versorgungsforschung

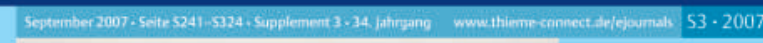

Multiplizität der

Depression

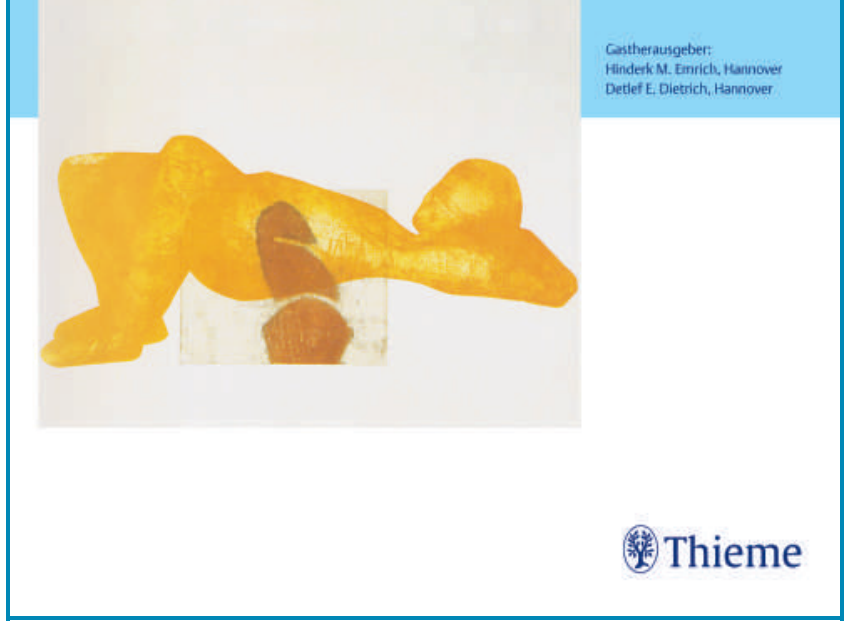

Psychiat Prax 2007, Sonderheft 3:

Leider wurde der Name der Künstlerin versehentlich nicht abgedruckt.

Unser Titelbild stammt von Frau Claudia Schmidt, Hannover, Farbradierung, „O.T.“, 1997. 